\title{
Divergencia empresarial en la productividad total de los factores en el período de crisis y recuperación económica en el sector turístico español
}

\author{
Albert Miró* \\ Universitat de Vic (España)
}

\begin{abstract}
Resumen: El objetivo principal de esta investigación consiste en contrastar la existencia de una relación positiva entre la productividad total de los factores (PTF) de las empresas del sector turístico español y la utilización de las Tecnologías de la Información y la Comunicación (TIC). Entendiendo que las empresas que optan por la inversión y el desarrollo de las TIC mejoran su PTF. Así como la interacción con el mercado internacional (exportación e importación) conllevan a una mejora de la PTF en el contexto de la "New" new trade theory. Asimismo, el debate actual sobre la dispersión de la PTF ha afectado a todos los sectores económicos. En este caso la hipótesis planteada gira en torno a la existencia de una divergencia de dicha variable entre un período de crisis (2007-2011) y un período de recuperación económica (2012-2017). Para el correcto desarrollo de la investigación se han extraído los datos del Sistema de Análisis de Balances Ibéricos (SABI), que han permitido estimar la PTF mediante MCO, EF y LP, así como la correcta constatación de las hipótesis mediante el método SEM. Tres conclusiones se anuncian en este artículo: i) Se argumenta que el sector turístico español parece tener un bajo nivel de adopción de las TIC en su estructura empresarial; ii) si se demuestra una dispersión de la PTF con respecto a los dos subperíodos analizados (crisis y recuperación económica), y iii) la internacionalización tiene un resultado significativo en relación con la PTF.
\end{abstract}

Palabras Clave: España; Productividad total de los factores; Internacionalización; Turismo; Dispersión.

Business divergence in the Total Productivity of Factors in the period of crisis and economic recovery in the Spanish tourism sector

Abstract: The main aim of this research is to contrast the existence of a positive relationship between the total factor productivity (TFP) of companies in the Spanish tourism sector and their use of Information and Communication Technologies (ICT). in the understanding that the trend is for companies to opt for ICT investment and development to improve their TFP and their interaction with the international market (export and import) also leads to an improvement in TFP in the context of the "New" new trade theory.

Likewise, the current debate on the dispersion of TFP has affected all economic sectors. In this case, the hypothesis revolves around the existence of a divergence of this variable between a period of crisis (2007-2011) and a period of economic recovery (2012-2017). The data from the Iberian Balance Sheet Analysis System (SABI) were extracted fto the ends of the correct development of this research which has allowed the TFP to be estimated using MCO, EF and LP, as well as the correct verification of the hypotheses using the SEM method. Three conclusions are reached: i) that the Spanish tourism sector seems to have a low level of ICT adoption in its business structure; ii) that TFP dispersion is demonstrated with respect to the two subperiods analysed (crisis and economic recovery), and that iii) internationalisation has a significant result on TFP.

Keywords: Spain; Total factor productivity; Internationalisation; Tourism; Dispersion.

\section{Introducción}

En los últimos años la realidad de la literatura económica actual ha identificado el nuevo paradigma imperante, que tiene como base tres pilares fundamentales: el proceso de mundialización, la

Universitat de Vic (España); E-mail: albertpol.miro@uvic.cat 
revolución tecnológica industrial y los cambios en los patrones de demanda y las empresas (Pérez, 2010; Drucker, 2012).

La irrupción de las nuevas tecnologías digitales ha transformado de manera notable la actividad económica. Éstas se agrupan bajo el paraguas de las tecnologías de la información y la comunicación (TIC), las cuales integran un conjunto convergente de tecnologías que se aplican de manera adecuada en la esfera económica (Vu, 2011; Jorgenson y Vu, 2016).

La utilización masiva de las TIC, en las cuales se incluyen diferentes recursos como son ordenadores, líneas telefónicas digitales, internet, redes de comunicación, correo electrónico, teléfono móvil, entre otras, ha permitido generar una transformación de los usos productivos actuales (Luintel et al., 2014). Bajo este prisma se desprende un incremento de la competencia, un cambio organizacional, así como patrones de eficiencia que determinan el trabajo (Roth y Thum, 2013). La nueva economía conlleva que los flujos de información y conocimiento se conviertan en los determinantes de los avances de productividad y competitividad (Torrent-Sellens, 2004; Torrent-Sellens, 2016).

Es por ello que la incorporación de la tecnología ha provocado una nueva aproximación metodológica que la literatura ha debatido sobre la evidencia de la existencia causal entre la agregación de este nuevo recurso y una mejora de la productividad y la eficiencia empresarial (O'Mahony y Vecchi, 2003; Roth y Thum, 2013). Así, la literatura económica ha introducido el concepto de heterogeneidad empresarial en el análisis del ciclo de vida, condicionado por la capacidad productiva de cada una de las empresas (Bernard et al., 2010; Syverson, 2011)

El análisis del cambio disruptivo de los sectores económicos ha sido global, y en este ambiente se ha producido una notable evolución del sector turístico. La penetración de las TIC en dicho sector ha sido significativo en los últimos años (Hjalager, 2010; Law, Buhalis y Cobanoglu, 2014). Además, otros niveles parecen ser relevantes, como la adopción de tecnologías inteligentes (IoT, Big Data, Inteligencia Artificial, etc.) (Trequattrini, Shams, Lardo y Lombardi, 2016; Lombardi, 2019).

En las últimas décadas el sector turístico español ha ganado relevancia de manera notable (Esteban Talaya et al., 2000; Alberca y Parte, 2013; Cànoves et al, 2016), lo que ha llevado a la necesidad de observar el impacto que tienen las nuevas variables (adopción de tecnologías inteligentes, capital humano preparada, inversión en TIC, entre otros) en el crecimiento a un futuro inmediato, entendiéndose dichas variables como la productividad total de los factores (PTF). En este caso el análisis que se plantea en este artículo se focaliza en el sector turístico español para el período de tiempo comprendido entre los años 2007 al 2017.

La incorporación del conocimiento al conjunto de ramas productivas de la economía nacional ha permitido aproximar la economía del conocimiento al tejido industrial nacional. Es por ello que los principales objetivos de este trabajo vienen determinados por el análisis de la transformación de la actividad económica del sector turístico español de una economía tradicional hacia una economía basada en la economía del conocimiento, tanto a nivel agregado (por país) como desagregado por Comunidad Autónoma (CC.AA). Así como, analizar dicho sector con respecto a la incorporación de las TIC por rama productiva.

En esta investigación se pretende dar respuesta a la hipótesis basada en la economía del conocimiento:

$H_{r}:$ La utilización de las TIC conlleva una mejora de la productividad empresarial.

$H_{2}$ : la utilización de las páginas web tiene un efecto positivo en la PTF.

Bajo la misma línea de investigación se plantea un segunda hipótesis se asocia a la "New" new trade theory. El cual se asocia al incremento del comercio intra-industrial relajando los supuestos de competencia perfecta. En este sentido se evidencian las pautas de la internacionalización de las empresas (Helpman et al., 2004; Wagner, 2007).

\section{$H_{3}:$ Existe una relación causal entre la internacionalización de la empresa con respecto a la PTF.}

Además, se pretende ver si existe una relación directa entre la divergencia de la PTF con respecto a dos períodos diferenciados como son la pre-crisis (del año 2007 al 2011) y la post-crisis (del año 2012 al 2017). Definiendo una tercera hipótesis como:

\section{$H_{4}:$ Hay una dispersión de la variable PTF con respecto al período temporal analizado.}


El resto de trabajo se organiza como sigue. En el segundo epígrafe se realiza una revisión de la literatura que analiza el sector turístico español, la productividad total de los factores, así como el análisis de la implantación de las TIC en las empresas turísticas como variable competitiva.

En el tercer apartado se presenta la metodología utilizada para realizar las correspondientes estimaciones. En el cuarto epígrafe se presenta la base de datos utilizada para la medición de PTF y la relación existente entre esta variable y la TICs. En la quinta sección, se analizan los principales resultados obtenidos. Y, por último, se realiza una discusión de los resultados, así como se realiza una propuesta de unas breves conclusiones.

\section{Marco teórico}

Desde la última década la actividad económica se encuentra inmersa en un proceso de constante transformación (Cristelli et al., 2015). Si bien los fundamentos de esta dinámica son múltiples, existe un consenso en la literatura que atribuye este proceso a una triple interacción considerada como el proceso de la revolución tecnológica, con una mayor utilización de las TIC; el proceso de la globalización 2.0, y por último, el cambio del patrón de la demanda de consumo y de la inversión de familias y empresas (Castells, 2004; Vilaseca y Torrent, 2005).

Así, la revolución digital, con la incorporación masiva de las TIC, ha provocado una clara modificación en todas las facetas de la vida humana, favoreciendo una apertura hacia nuevas oportunidades de crecimiento y desarrollo económico (Johnson, 2018). Por tanto, esta economía del conocimiento ha llevado a una nueva revolución industrial, con una nueva articulación económica y social asociada, donde los flujos de información y conocimiento se convierten en los determinantes de los avances de productividad y competitividad (O’Mahony y Vecchi, 2003).

Durante la última década la economía internacional ha vivido una crisis lo que se ha traducido en un incremento en la literatura interesada en aquellos factores que afectan a la PTF (Mokyr, 2014; Autor, 2015; Graetz y Michaels, 2017). En este sentido se ha debatido mucho sobre el peso preponderante que han tenido las TIC en la actividad productiva, y por ende, en las capacidades de las PTF (Brynjolfsson y Hitt, 2000); detectándose una relación positiva entre una mayor inversión en TIC y una mejora en la productividad (Brynjolfsson y Hitt, 1996, 2000, 2003; Dedrick et al., 2003)

Así mismo, este crecimiento ha provocado un renovado interés en el análisis de las diferencias entre los resultados económicos de los países y la relación de dichas diferencias en el dispersión de la productividad dentro de la industria a través de las empresas (Andrews, Criscuolo y Gal, 2015; Andrews, Criscuolo y Gal, 2016; Añon Higón et al., 2017). También se ha señalado como existe una dispersión de la productividad dentro de los propios países (Ito y Lechevalier, 2009; Iacovone y Crespi, 2010; Berlingieri, Blanchenay y Crisuolo, 2017).

En cuanto al impacto de las TIC en la industria manufacturera se ha realizado una gran actividad investigadora (Gust y Marquez, 2004; Giuri et al., 2008; Andrew et al., 2015). Si bien cabe destacar que el impacto del estudio de esta relación en el sector servicio es menor (Bernard y Jones, 1996; Brynjolfsson y Yang, 1996), aún lo es más en la literatura que hace referencia al sector turístico (Buhalis y Law, 2008; Li y Prescott, 2009; Kumar y Singh, 2014).

El sector turístico se caracteriza por un uso intensivo de la información, es por ello que viene determinado por un notable impacto de las TIC en dicha actividad turística (Ivars, Solsona y Giner, 2015).

Concretamente, en España el sector turístico tiene una gran impronta en la economía nacional, siendo uno de los motores de crecimiento económico por su aportación al PIB nacional, así como por su capacidad de generación de renta y empleo (Roura y Morales, 2011; Gómez, 2015). Parte importante de la novedad aportada por este artículo viene asociada por la mejora de la aportación a la relación existente entre las TIC y la PTF a nivel del sector turístico, si bien existe una literatura que trata dicha relación se basa en sector hotelero (Benavides, 2012; Alberca y Parte, 2010; Alberca y Parte, 2013; Cerezo y Guevara, 2015) o a nivel más agregado del sector turístico (Miró y Pereira, 2017).

De igual manera, el tejido del sector turístico español se constituye mayoritariamente por pequeñas y medianas empresas (PyMEs) que destacan por encima de las grandes empresas. En este sentido, cabe remarcar como la utilización de las TIC permite a las PyMEs incidir en una mejora en la productividad (UNCTAD, 2008; Díaz-Chao et al., 2015). No obstante, las PyMEs tienes un menor grado de apertura al mercado internacional, así como en la entrada de innovación (Drechsler y Natter, 2012). El tejido 
industrial español queda conformado con un total del 99.88\% de PyMEs (Iglesias, Jambrino y De las Heras, 2017).

En base a esta afirmación, Sánchez (2017) declara como una baja calidad del capital humano tiene una relación causal con una reducida capacidad de innovación por parte de las empresas. Es por ello que en el conjunto de la Teoría de la empresa basada en el conocimiento se constata la necesidad de afirmar que el conocimiento se ha convertido en un recurso intangible de la mayor importancia para competitividad de las empresas (Tseng y Goo, 2005; Delgado-Verde et al., 2011).

En este caso, hay que tener en cuenta como una mejora de las políticas públicas en cuanto a la inversión en I + D no ha producido un efecto positivo esperado. Esta realidad puede deberse tanto al capital humano (tal como se ha destacado anteriormente) como a la estructura de tamaño de las empresas del tejido industrial español donde predominan las PyMEs que conduce a una menor productividad media de los trabajadores (Costa, 2015; Sánchez, 2017) y que se encuentra íntimamente ligado al concepto de innovación (Wu et al., 2008; Martin et al., 2009).

La bibliografía sobre esta temática ha debatido ampliamente mostrando que "el establecimiento de relaciones de complementariedad entre el capital humano, la reorganización organizativa y los usos de las TIC, se configura como un potente determinante en la explicación de la ventaja competitiva de las empresas"(Torrent-Sellens y Ficapal-Cusí, 2010: 205). Ante esta realidad se afirma que la creación de valor y la concepción de una nueva ventaja competitiva hacia las empresas viene determinado por una mayor contribución de aquellos elementos que vienen determinados como una naturaleza inmaterial, o también conocido como activos intangibles (Díaz-Chao y Torrent-Sellens, 2010).

Bajo este prisma se puede afirmar que la competitividad española queda afectada de manera negativa. En cuanto al capital humano la literatura de la teoría económica mantiene que este influye de manera positiva en el desarrollo económico, así como en el crecimiento de la productividad (Ciccone y Paoaioannou, 2005). Aun así, España presenta ciertas limitaciones en la inversión en capital humano que limita la generación de un proceso positivo en cuanto a la innovación (Zayas, 2015).

Si bien la crisis internacional acaecida a partir del 2007 ha conllevado un freno al crecimiento medio asociado a la productividad (Renda y Dougherty, 2016). Bajo esta circunstancia la literatura constata como a partir de dicho período se ha generado la "gran divergencia" (Berlingieri et al., 2017). Este concepto viene ligado directamente a la disparidad generada a partir de los años 2007-2008 entre el $10 \%$ de las empresas más productivas del sector manufacturero y servicios (las conocidas como empresas frontera) y el 90\% de empresas restantes (Bartelsman et al., 2013; Andrews et al., 2015; Berlingieri et al., 2017; Añon et al., 2017). Esta circunstancia es debido a que las empresas frontera son más intensivas en capital, mejores habilidades laborales, y mayor inversión en nuevas tecnologías e innovación (Iacovone y Crespi, 2010; Van der Wiel et al., 2012; Añon et al., 2017). Es por ello que el deterioro precisamente del capital fue el responsable del decremento de la PTF de los años comprendidos entre el 1995 y el 2007 (García-Santana et al., 2016; Fu y Moral-Benito, 2018). No obstante, hubo un incremento en la inversión en capital a partir del año 2007 que permitió una mejora de la PTF, si bien la productividad laboral también tuvo una mejora a partir del inicio de la crisis (Fu y Moral-Benito, 2018).

\section{Metodología}

A fin de analizar la PTF de las empresas que conforman el sector turístico español para los años comprendidos entre el 2007 al 2017 es importante definir ambas variables, así como la base de datos de la cual se extrae la información necesaria para contrastar las hipótesis determinadas.

\subsection{Productividad Total de los Factores}

La estimación de la PTF se puede realizar a partir de la función de producción Cobb-Douglas, donde el output es el producto de una función de inputs observables y de un factor-neutral en el sentido de Hicks.

$$
Y_{i t}=A_{i t} F\left(K_{i t}, L_{i t}, M_{i t}\right)
$$

Donde $Y$ es el producto, y $F(\cdot)$ es la función de inputs observables que se supone que es continua y univoca. Así como, los inputs son $K$ es el coste de capital, $L$ el de trabajo, $M$ es el coste intermedio (Melitz, 2000) y $A$ es la productividad total de los factores o nivel de eficiencia neutral hicksiano. El 
subíndice $i$ representa a la i-ésima empresa y $t$ es período de tiempo comprendido entre $\mathrm{t}=2007, \ldots$, 2017. La función [1] se puede reescribir de forma algebraica en,

$$
Y_{i t}=A_{i t} L_{i t}^{\beta_{l}} K_{i t}^{\beta_{k}} M_{i t}^{\beta_{m}}
$$

La estimación de la PTF a nivel empresa se realiza a partir de la siguiente ecuación.

$$
y_{i t}=\beta_{0}+\beta_{l} l_{i t}+\beta_{k} k_{i t}+\beta_{m} m_{i t}+u_{i t}
$$

Donde $u$ representa el término de error, el cual recoge todos aquellos factores de la realidad no observables o los debidos a circunstancias externas. $\beta_{0}$ que es común en todas las unidades de producción en la muestra (por lo general la tecnología se estima en el nivel de la industria).

Para la obtención de la medición de la PTF se obtiene con respecto a la función [4], así mismo se puede evaluar la influencia de las diferentes variables en la empresa.

$$
\ln \left(A_{i t}\right)=y_{i t}-\widehat{\beta}_{l} l_{i t}-\widehat{\beta_{k}} k_{i t}-\widehat{\beta_{m}} m_{i t}
$$

Para la estimación de la PTF se han utilizado tres enfoques metodológicos. El primero es el Mínimo Cuadrado Ordinario (MCO), el de Efectos Fijos (EF) y Levinsohn y Petrin (2000) (LP). Los dos primeros métodos pueden tener problemas de endogeneidad que pueden existir entre los factores productivos. Cabe mencionar que el método de LP recurre a la utilización de una proxy para los shocks de productividad no observados con los inputs intermedios, ya que la utilización de la inversión como proxy conlleva el siguiente problema: la inversión que es una variable muy desigual (lumpy) debido a que incorpora costes de ajuste sustanciales y por lo tanto, no responde "suavemente" a choques en productividad.

Por último, una vez planteado el tema de la productividad individual queda pendiente qué factores implican un crecimiento de la productividad agregada. Existen dos efectos complementarios que muestran dicho aumento: el crecimiento de las productividades individuales, y la redistribución de los inputs entre compañías, que llevan a una mejora de la productividad global.

Una primera aproximación a este efecto de productividad agregada viene determinado por Foster, Haltiwagner y Krizan (1998) que muestran que el crecimiento de PTF de un período $t$ a otro $t$ - 1 viene determinado por,

$$
\ln P T F_{t}-\ln P T F_{t-1}
$$

El crecimiento de la PTF, por tanto, compara diferentes puntos o elementos en el tiempo, mientras que el nivel de PTF, como se ha podido observar, compara estos elementos en el espacio.

\subsection{Modelo de ecuación estructural}

La metodología propuesta para probar la hipótesis es un modelo de ecuación estructural. Este método permite encontrar relaciones directas entre las variables del modelo mientras se analiza el impacto entre las construcciones. El modelo SEM permite realizar una estimación entre variables continuas como dependientes y variables independientes que pueden ser continuas, binarias, y/o categóricas.

En el siguiente modelo (Figura 1) se indica como la variable dependiente es PTF, asimismo se analiza la supuesta relación existente con respecto a las variables independientes:

- Gasto en I+D (Lntic).

- Realiza exportación, queda definida como variable dicotómica que toma valor 1 si realiza actividad exportadora y 0 en caso contrario (Export).

- Realiza importación, en este caso vuelve a ser una variable dicotómica la cual toma valor 1 si realiza importación y 0 si no realiza dicha actividad (Import).

- Tiene página web. Donde 1 tiene página web y 0 no tiene página web (WP). 


\section{Figura 1: Modelo de Ecuaciones Estructurales inicial}

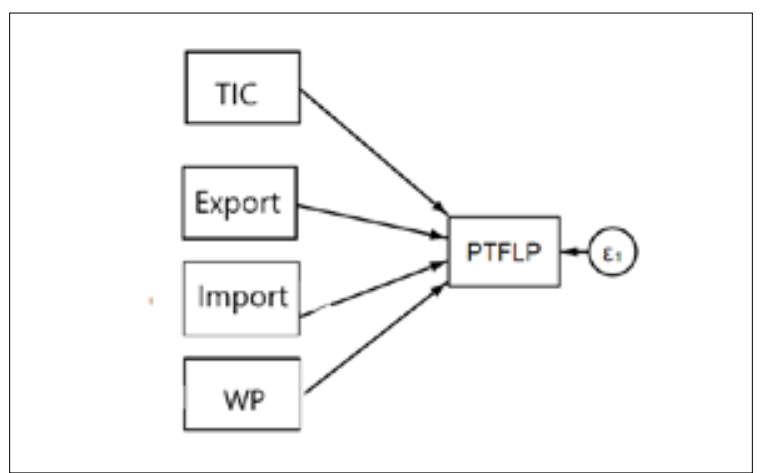

Fuente: Elaboración propia

\subsection{Base de datos}

Para cumplir con los objetivos propuestos se empleó la información de la base de datos Sistema de Análisis de Balances Ibéricos (SABI), resultado de la colaboración de tres estamentos privados, Informa D\&B, Coface Serviços Portugal, S.A y Bureau Van Dijk. La disponibilidad de información de tipo microeconómica es fundamental para establecer los mecanismos que condicionan el desempeño de las empresas. Por tanto, trabajar con esta base de datos permite desagregar la información sobre las características de las empresas, lo cual ha proporcionado que parte de la metodología utilizada en esta investigación haga uso de técnicas de la estadística descriptiva y de los modelos de la econometría (Coll-Serrano y Blasco-Blasco, 2009; Fernández de Guevara, 2011; Miró-Pérez, 2018).

Una de las cuestiones que se plantea en este y otros trabajos es el tratamiento para transformar las variables de nominales a reales, sin el efecto derivado de la variación de los precios. Por esta razón, las variables quedarán deflactadas utilizando el deflactor de los índices de precios al consumo para corregir los precios - estos datos se han sonsacado del Instituto Nacional de Estadística (ine.es)- y la limitación el impacto de los mismos en la medida de la producción de la empresa y en las estimaciones de la función de producción (De Loecker, 2007).

A continuación se muestra un resumen de las variables dependientes y explicativas incluidas en los modelos utilizados a nivel de productividad (Tabla 1). Estas variables quedan justificadas en base a la literatura que trata la estimación de la PTF.

Tabla 1: Variables

\begin{tabular}{|l|l|}
\hline \multicolumn{1}{|c|}{ Variables* } & \multicolumn{1}{c|}{ Bibliografia } \\
\hline Dependiente & \\
\hline Ingreso de explotación & Olley y Pakes (1996) \\
\hline Independientes & \\
\hline Activo intangible & Díaz-Chao y Torrent-Sellens (2010) \\
\hline Capital & Olley y Pakes (1996) \\
\hline Coste Material & Levisohn y Petrin (2004) \\
\hline Coste Laboral & Olley y Pakes (1996) \\
\hline
\end{tabular}

*En este caso dichas variables se tratan en la medida de euros.

Fuente: Elaboración propia

La delimitación de las actividades económicas características del turismo ha sido identificada en diversas recomendaciones de organismos internacionales como la Organización Mundial de Turismo (OMT) y la Comisión de Estadística de Naciones Unidas. Para ello se seleccionan en base al código CNAE-2009 aquellas actividades que se consideran actividades asociadas al sector turístico (Tabla 2). 
Tabla 2: Actividades CNAE-2009 asociadas al sector turístico (\%)

\begin{tabular}{|l|l|c|}
\hline CNAE-2009 & \multicolumn{1}{|c|}{ Actividad económica } & Frecuencia \\
\hline 55 y 56 & Hostelería & 30.13 \\
\hline 491 & Transporte interurbano de pasajeros por ferrocarril & 0.25 \\
\hline 493 & Otro transporte terrestre de pasajeros & 11.56 \\
\hline 501 & Transporte marítimo de pasajeros & 1.01 \\
\hline 511 & Transporte aéreo de pasajeros & 1.53 \\
\hline 522 & Actividades anexas al transporte & 28.90 \\
\hline 791 & Actividades de agencias de viajes y operadores turísticos & 11.54 \\
\hline 799 & Otros servicios de reservas y actividades relacionadas con los mismos & 0.25 \\
\hline 771 & Alquiler de vehículos de motor & 3.77 \\
\hline 773 & Alquiler de otra maquinaria, equipos y bienes tangibles & 2.76 \\
\hline 900 & Actividades de creación, artísticas y espectáculos & 1.01 \\
\hline 910 & Actividades de bibliotecas, archivos, museos y otras actividades culturales & 0.75 \\
\hline 931 & Actividades deportivas & 5.78 \\
\hline 932 & Actividades recreativas y de entretenimiento & 0.75 \\
\hline
\end{tabular}

Fuente: Elaboración propia

Por último, en referencia al volumen de trabajadores por empresa la clasificación viene determinado por el conjunto de microempresas (0-9 asalariados), pequeñas (10-49 asalariados) y medianas empresas (50-199 asalariados) que conforman el grupo de las PyMEs, y grandes empresas (a partir de 250 asalariados). En este caso, se definen cuatro agrupaciones de empresas (microempresa, pequeña, mediana y gran empresa) tomando como criterio únicamente el número de empleados. Cabe destacar que estas agrupaciones se han ajustado a la recomendación de la Comunidad Europea del 6 de mayo de 2003 (DOC (2003) 1422).

Con respecto a la tendencia de los diferentes grupos con respecto al tamaño empresarial en los años objeto de estudio se muestran en la siguiente Figura 2, en la cual en base al año base es el 2007 podemos observar como existe una claro decremento de las medianas empresas con un valor aproximado del $55 \%$ de pérdidas de empresas a lo largo de la muestra, mientras se crean un mayor número medio de micro y pequeñas empresas, con un $17 \%$ y $7 \%$, respectivamente. En cuanto a las grandes empresas también se constata un claro crecimiento, con un valor cercano al $6 \%$.

Figura 2: Tendencia del tamaño empresarial. Años 2007-2016 (año base 2007)

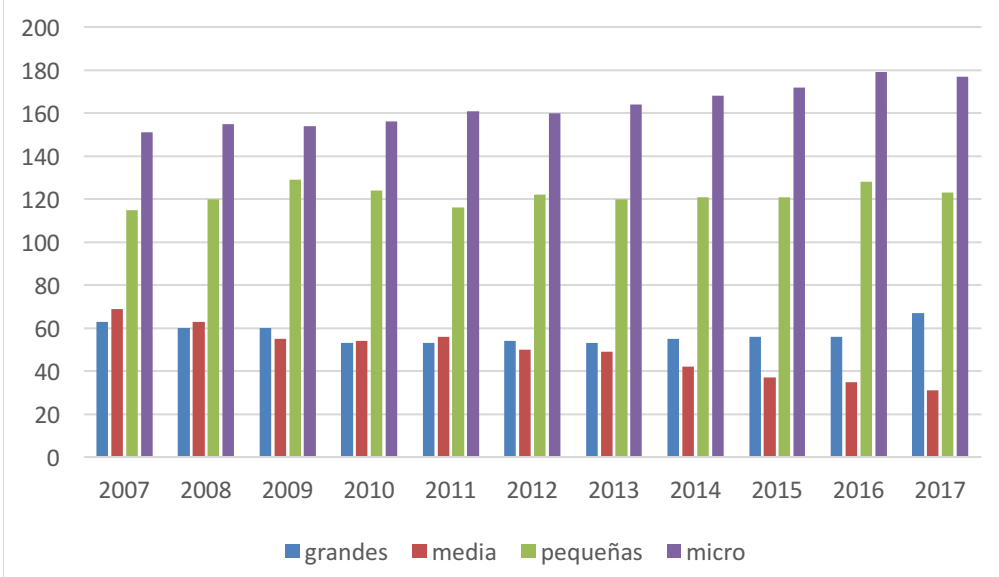

Fuente: Elaboración propia 
Es por ello que SABI permite extraer los datos necesarios para las muestras deseadas, y también amplía de manera considerable los aspectos que engloban la pequeña y mediana empresa (PyMEs) y las grandes empresas que pueden ser sometidas a investigación.

\section{Resultados}

\subsection{Productividad Total de los Factores}

El primer ejercicio verifica la estimación de la PTF utilizando tres metodologías como son LP, MCO y EF. En la Tabla 3 se muestra el resumen de los resultados obtenidos de las estimaciones del modelo por dichas metodologías.

Los resultados obtenidos muestran que los parámetros son estadísticamente significativos y los signos esperados, son todos positivos excepto la variable TIC con respecto a la estimación con LP y EF. Con respecto al resultado obtenido cabe destacar como la TIC tiene una relación positiva hacia la mejora de los Ingresos de Explotación de las empresas con respecto a la estimación de LP y MCO, y una relación negativa con la EF. Así mismo, los resultados obtenidos por LP muestran un resultado no significativo, en cambio con MCO y EF son significativos. Cabe destacar como de manera independiente a la metodología utilizada se puede observar que bajo condiciones de cetearis paribus, las elasticidades de los factores productivos son bajas.

Tabla 3: Cuadro resumen de la estimación por LP, MCO y EF

\begin{tabular}{|c|c|c|c|}
\hline Variable & LP & MCO & EF \\
\hline $\operatorname{lnCm}$ & $\begin{array}{l}0.261^{*} \\
(0.000)\end{array}$ & $\begin{array}{l}0.254^{*} \\
(0.000)\end{array}$ & $\begin{array}{l}0.225^{*} \\
(0.000)\end{array}$ \\
\hline $\operatorname{lnCm}$ & $\begin{array}{l}0.428^{*} \\
(0.000)\end{array}$ & $\begin{array}{l}0.471^{*} \\
(0.000)\end{array}$ & $\begin{array}{l}0.593^{*} \\
(0.000)\end{array}$ \\
\hline lnCapital & $\begin{array}{l}-0.053^{*} \\
(0.015)\end{array}$ & $\begin{array}{l}0.015^{*} \\
(0.007)\end{array}$ & $\begin{array}{c}0.012^{* * * *} \\
(0.098)\end{array}$ \\
\hline $\operatorname{lnTIC}$ & $\begin{array}{r}0.012 \\
(0.149)\end{array}$ & $\begin{array}{c}0.012^{*} \\
(0.032)\end{array}$ & $\begin{array}{c}-0.007 * \\
(0.002)\end{array}$ \\
\hline
\end{tabular}

Nota: $* * * /$ significativo al $10 \%, * * /$ significativo al $5 \%, * /$ significativo al $1 \%$

Fuente: Elaboración propia

Los indicadores de tiempo se dividen en una variable dummy donde toma el valor 0 para el periodo de tiempo pre-crisis que corresponde a los años 2007-2011, y el valor 1 para el período post-crisis del 2012-2017 (ver Tabla 4). Los resultados obtenidos a partir del test de normalidad de Kolmogorov-Smirnov con respecto a la variable dependiente PTF no muestra una clara diferencia entre el cambio de tendencia de PTF con respecto al cambio de periodo temporal.

Así pues, en base al resultado obtenido la hipótesis nula queda refutada al nivel de 0.113. Este resultado muestra como los valores observados de las frecuencias para cada clase o grupo no demuestran la existencia de una diferencia de la frecuencia teórica de una distribución normal.

Tabla 4: Test Kolmogorov-Smirnov

\begin{tabular}{|l|c|c|c|}
\hline \multicolumn{1}{|c|}{ Smaller Group } & D & P-Value & Corrected \\
\hline $0:$ & 0.0491 & 0.062 & \\
\hline $1:$ & -0.0083 & 0.924 & \\
\hline Combined K-S: & 0.0491 & 0.124 & 0.113 \\
\hline
\end{tabular}

Fuente: Elaboración propia 
La siguiente Tabla 5 muestra las diferencias medias de la PTF de manera agregada al periodo objeto de estudio, así como desagregado mediante los períodos pre y post-crisis. En todos los casos, las diferencias en las medias entre empresas fronterizas -definidas como aquellas empresas que tienen un valor más elevado de PTF- y no fronterizas -empresas con valores medios de PTF bajos- se basan en la clasificación según la medida de productividad de la PTF.

\section{Tabla 5: Media y diferencias en media de las características de las empresas: Empresas frontera vs. No frontera}

\begin{tabular}{|l|c|c|}
\cline { 2 - 3 } \multicolumn{1}{c|}{} & \multicolumn{2}{c|}{ PTF } \\
\cline { 2 - 3 } \multicolumn{1}{c|}{} & NF & F \\
\hline Total años: $2007-2017$ & 3.879 & 5.465 \\
\hline Pre-crisis: 2007 & 3.855 & 5.445 \\
\hline Post-crisis: 2011 & 3.888 & 4.571 \\
\hline
\end{tabular}

Nota: Para identificar la Frontera, se ha calculado el número medio de empresas a través de los años para el sector turístico, y el 5\% de este número medio es el número de empresas que se consideran constantes a través del periodo objeto de estudio que se identifican como las empresas más productivas. Así, F es el promedio de empresas frontera, y NF es el promedio de empresas no frontera (el resto de empresas).

Fuente: Elaboración propia

Durante el período comprendido entre 2007 y 2017, las empresas en la frontera del sector turístico español son, en promedio, una vez más productivas que las empresas no fronterizas, lo que indica que la brecha de PTF entre las empresas fronterizas y no fronteriza no se debe considerar como muy dispar y, por lo tanto, sugiere que el potencial de captura de mejora de PTF sigue siendo elevado, pero menor de lo esperado.

Al comparar las estadísticas reportadas para el año 2007 (recesión) y el año 2011 (recuperación), se observa que hay una reducción en la PTF promedio de las empresas tanto fronterizas como las no fronterizas.

En la siguiente Tabla 6 se resumen los resultados de las principales actividades empresariales del sector turístico definido por su código CNAE-2009 con respecto a la medida de PTF. En la primera columna se determina el código CNAE-2009. En la segunda los valores medios de PTF en época de recesión, y en la tercera se definen los valores de la etapa de recuperación económica. Por último, en la cuarta columna se encuentra la media por el total de años estudiados.

Este supuesto permite categorizar según aquellas actividades con un valor medio más elevado, en este caso Otros servicios de reservas y actividades relacionadas con los mismos (799), Actividades anexas al transporte (522), y Alquiler de vehículos de motor (771) son las que tienen un valor mayor. Mientras que Hostelería (55 y 56) es aquella que tiene valores medios menores. Existe una diferencia notable entre el primer y el último valor cercano al $55 \%$.

Cabe destacar como existen muchas actividades que tenían un valor medio PTF más elevado en época de recesión, tales como son Alquiler de vehículos de motor (7711), Actividades de creación, artísticas y espectáculos (9001), Actividades de bibliotecas, archivos, museos y otras actividades culturales (9104). No obstante, las empresas que constituyen un valor medio mayor en época de recuperación son de un $50 \%$. En época de recuperación, el sector turístico ha realizado una inversión en TIC elevada, y a su vez España ha incrementado el número de turistas en los últimos años. 
Tabla 6: PTF por CNAE-2009

\begin{tabular}{|c|c|c|c|c|c|c|c|}
\hline CNAE & Recesión & Recuperación & $\begin{array}{l}\text { Total } \\
\text { años }\end{array}$ & CNAE & Recesión & Recuperación & $\begin{array}{l}\text { Total } \\
\text { años }\end{array}$ \\
\hline 7990 & 4.986 & 5.036 & 5.021 & 5222 & 3.800 & 3.948 & 3.907 \\
\hline 5221 & 4.614 & 4.734 & 4.706 & 5590 & 3.676 & 3.867 & 3.810 \\
\hline 7711 & 4.654 & 4.609 & 4.621 & 9312 & 3.690 & 3.837 & 3.808 \\
\hline 5110 & 4.467 & 4.617 & 4.574 & 7735 & 3.882 & 3.769 & 3.803 \\
\hline 5224 & 4.446 & 4.515 & 4.497 & 4910 & - & 3.778 & 3.778 \\
\hline 9001 & 4.573 & 4.285 & 4.400 & 5510 & 3.695 & 3.788 & 3.765 \\
\hline 5010 & 4.401 & 4.390 & 4.393 & 9319 & 3.142 & 3.942 & 3.764 \\
\hline 7911 & 4.177 & 4.405 & 4.346 & 9004 & 3.784 & 3.698 & 3.718 \\
\hline 7912 & 4.196 & 4.390 & 4.333 & 5630 & 3.642 & 3.751 & 3.718 \\
\hline 7733 & 4.430 & 4.109 & 4.204 & 5610 & 3.637 & 3.735 & 3.709 \\
\hline 9104 & 4.442 & 4.117 & 4.163 & 4939 & 3.706 & 3.704 & 3.704 \\
\hline 9321 & 4.048 & 4.172 & 4.144 & 7732 & 3.759 & 3.676 & 3.697 \\
\hline 9311 & 3.920 & 4.246 & 4.142 & 4931 & 3.678 & 3.646 & 3.654 \\
\hline 5229 & 4.017 & 4.091 & 4.072 & 5520 & 3.504 & 3.615 & 3.593 \\
\hline 5223 & 3.968 & 3.993 & 3.987 & 5629 & 3.571 & 3.569 & 3.570 \\
\hline 7739 & 3.897 & 3.988 & 3.967 & 5621 & 3.500 & 3.473 & 3.480 \\
\hline 9329 & 3962 & 3.945 & 3.949 & 5530 & - & 3.234 & 3.234 \\
\hline 9313 & - & 3.920 & 3.920 & & & & \\
\hline
\end{tabular}

Fuente: Elaboración propia

Cabe esperar que las empresas de gran tamaño tengan un valor medio de PTF mayor con respecto a las PyMEs. No obstante, los principales valores descriptivos obtenidos en la Tabla 7 muestran como son las Pequeñas y microempresas aquellas que tienen un valor de PTF más elevada. Puede observarse como existe una variación de la microempresa con respecto a la mediana y gran empresa del $2.66 \%$ y $7.79 \%$, respectivamente. Mientras que la variación de la pequeña empresa con la mediana y gran empresa corresponde al $4.63 \%$ y el $9.86 \%$, respectivamente.

Tabla 7: PTF por cohorte de tamaño empresarial

\begin{tabular}{|l|c|}
\hline Cohorte & PTF \\
\hline Microempresa & 4.165 \\
\hline Pequeña empresa & 4.245 \\
\hline Mediana empresa & 4.057 \\
\hline Gran empresa & 3.864 \\
\hline
\end{tabular}

Fuente: Elaboración propia

\subsection{Método SEM}

En la siguiente Tabla 8 es muestran los principales resultados (coeficientes estandarizados y los errores estándar) de la estimación SEM para las empresas del sector turístico español, seguimos los indicadores minimos necesarios para demostrar la robustez del modelo (Schemelleh-Engel et al., 2003). 
Los resultados obtenidos muestran como en referencia a la $\mathrm{H} 1$ que indica la relación causal y positiva existente entre inversión en TIC y PTF, la cual muestra dicha relación. Esta correlación se constata en lo dicho anteriormente en la literatura en diferentes sectores económicos (Brynjolfsson y Hitt, 2003; Blooom et al., 2015), si bien existen menos análisis empíricos que aplican dicha hipótesis (Díaz-Chao et al., 2015).

En cuanto a la hipótesis de la importancia que tienen la utilización de la página web en el contexto de la mejora de la PTF vemos como el resultado es no significativo. Por tanto, es un resultado contrario a lo mostrado por la literatura (Miró y Pereira, 2017).

Por último, En cuanto a la hipótesis sobre internacionalización (H3 exportación y H4 importación) se constata como existe una relación significativa y positiva entre exportación y PTF, dentro de la "New" new trade theory el resultado es coherente mostrando como existe una relación de mejora entre la posición de exportación y la mejora de la PTF por aprendizaje (Aw, Chung, Roberts, 2000; Mengistae y Pattillo, 2004; De Loecker, 2013).

En cambio, la relación no es significativa en cuanto a la importación y la mejora de la PTF, rechazando dicha hipótesis esta situación se encuentra en conjunción con la realidad de la literatura de la Learning-by-importing (Vogel y Wagner, 2010).

Tabla 8: Factores explicativos de la PTF para las empresas del sector turístico español.

\begin{tabular}{|c|c|c|}
\hline Estructural: PTF & \multicolumn{2}{|c|}{ Coef. } \\
\hline TIC & \multicolumn{2}{|c|}{$\begin{array}{c}0.07^{* * *} \\
(0.000)\end{array}$} \\
\hline Export & \multicolumn{2}{|c|}{$\begin{array}{c}0.16^{* * * *} \\
(0.000)\end{array}$} \\
\hline Import & \multicolumn{2}{|c|}{$\begin{array}{c}0.02 \\
(0.538) \\
\end{array}$} \\
\hline WP & \multicolumn{2}{|c|}{$\begin{array}{c}0.02 \\
(0.808)\end{array}$} \\
\hline $\operatorname{Var}(\mathrm{e} . \mathrm{PTF})$ & \multicolumn{2}{|c|}{0.37} \\
\hline Índices & Valor & Valor Ideal \\
\hline Chi2_ms & 0.000 & \\
\hline RMSEA & 0.007 & $<0.06$ \\
\hline CFI & 0.970 & $>0.9$ \\
\hline TLI & 0.926 & $>0.95$ \\
\hline
\end{tabular}

Nota: Análisis de regresión: SEM con indicador latente y variables observables, y con medidas de error. Donde, ${ }^{* * *} \mathrm{p}<0.01 ;{ }^{* *} \mathrm{p}<0.05 ;{ }^{*} \mathrm{p}<0.1$.

RMSEA: Root Mean Square Error of Approximation; TLI: Tucker-Lewis Index; CFI: Comparative Fit Index. Fuente: Elaboración propia

\section{Discusión y conclusiones}

Existe todavía un gran número de problemas relacionados con la crisis que se dieron a partir del año 2007 y que afectaron a todos los sectores económicos a nivel internacional. Esta realidad también afectó de manera directa al sector turístico español. Pero ¿cómo ha afectado a la productividad empresarial? ¿Se ha dado una dispersión de dichas variables con respecto a la época de crisis y a la recuperación económica? En este artículo se ha pretendido dar respuesta a estas y otras cuestiones que afectan directamente a la competitividad nacional española de uno de sus sectores con más predominio en la economía nacional (Alberca, Parte y Such, 2011).

La estimación de la PTF nos ha permitido realizar una comparativa en la que se indica que bajo condiciones de cetearis paribus, las elasticidades de los factores productivos son bajas, resultado nada sorprendente en cuanto a la estimación de dichos factores para el cálculo de la PTF (Gómez, 2011). 
Así mismo, se ha querido determinar la influencia de la utilización de las TIC en cuanto al crecimiento de los ingresos de explotación. En los resultados obtenidos se muestra como existe una relación positiva con respecto a la variable dependiente y significativa con respecto a la estimación de MCO, mientras que para LP es no significativa.

Se argumenta que el sector turístico español parece tener un bajo nivel de adopción de las TIC en su estructura empresarial (Navio-Marco et al., 2018). Siguiendo evidencia de análisis previo. De esta manera, Miró sostiene que "los sectores económicos que desean participar de manera eficiente y ser competitivos en el mercado internacional, deberán avanzar en las TIC, ya que promueven cambios en el enfoque operativo, compras, negociaciones comerciales y comunicación" (Miró, 2018: 50) Por lo tanto, parece necesario analizar la PTF junto con la importancia del uso de las TIC.

Cabe destacar como en referencia a la dimensión o tamaño empresarial se demuestra cómo las pequeñas y micro empresas tienen un índice de la variable PTF mayor que las medianas y grandes empresas. Hay que indicar como el tejido industrial español y más concretamente, el sector turístico español en su mayoría son PyMEs, pero en este caso las empresas de mayor tamaño tienen una mejor PTF.

¿Cuáles son los subsectores más eficientes dentro del turismo? De nuestra evidencia, los grupos CNAE-2009 con el mejor valor de la PTF son Otros servicios de reservas y actividades relacionadas con los mismos (799), Actividades anexas al transporte (522), y Alquiler de vehículos de motor (771). Se constata como el sector alojamiento (55 y 56) es aquel con mayor número de empresas en el tejido turístico mostrando un amplio margen de mejora (Alberca y Parte, 2013).

Con respecto a las hipótesis planteadas se contrasta que no h ay una relación causal entre la utilización de las TIC con respecto a una mejora de la productividad. Por otro lado, si se demuestra una dispersión de la PTF con respecto a los dos sub-períodos analizados (crisis y recuperación económica).

El análisis llevado a cabo para contrastar las hipótesis ha permitido emerger las siguientes conclusiones asociadas a la relación significativa entre el uso de las TIC y la mejora de la PTF. Esta asociación se encuentra acorde con la literatura que indica como aquellas empresas que realizan inversión en TIC mejoran su productividad (Mohnen y Hall, 2013).

Así mismo, la internacionalización de las empresas en un contexto de heterogeneidad dentro del marco de la "New" new trade theory nuestras conclusiones referentes a la learning-by-exporting constatan una realidad palpable para el sector analizado en este artículo, y que se encuentran en que aquellas empresas que realizan exportación tienen una mejora consustancial de la PTF debido al aprendizaje que tienen una vez realizan actividad internacional (Wagner, 2007; Haidar, 2012; De Loecker, 2013).

Por último, la relación no es significativa en cuanto a la importación si bien la literatura de la learning-by-importing existe un gran debate sobre la impronta de la importación con respecto a la PTF, en este caso se constata que no existe una relación explicativa (Vogel y Wagner, 2010).

Sin embargo, la presente investigación presenta limitación. Esta viene asociada al análisis sobre la primera oleada de la digitalización, el cual incluye el internet no interactivo. No obstante, los resultados mostrados son adecuados con la literatura expuesta.

\section{Bibliografía}

Aigner, D., Lovell, C.A.L. y Schmidt, P. 1977. "Formulation and estimation of stochastic frontier production function models", Journal of econometrics, 6(1): 21-37.

Alberca, M.P., y Parte, L.P 2010. "Nuevas tecnologías y productividad en las empresas hoteleras: evidencia empírica (2000-2005)”, TuryDes, Revista de investigación en turismo y desarrollo local, 3(7), 1-20.

Alberca, M.P., Parte, L.P. y Such, M.J.S. 2011. "Evaluación del rendimiento de las empresas hoteleras con medidas de eficiencia y productividad: análisis frontera no paramétrico", Papeles de economía española, 128: 266-280.

Alberca, P. y Parte, L. 2013. "Evaluación de la eficiencia y la productividad en el sector hotelero español: Un análisis regional", Investigaciones Europeas de Dirección y Economía de la Empresa, 19: 102-111.

Andrews, D., Criscuolo, C. y Gal, P.N. 2015. "Frontier firms, technology diffusion and public policy: Micro evidence from OECD Countries", OECD productivity Working Papers, $\mathrm{N}^{\circ} 2$.

Andrews, D., Criscuolo, C. y Gal, P.N. 2016. "The Best versus the Rest: The Global Productivity Slowdown, Divergence across Firms and the Role of Public Policy", OECD Productivity Working Papers No. 5, OECD Publishing, Paris, DOI: https://doi.org/http://dx.doi.org/10.1787/63629cc9-en. 
Añon Higón, D., Mañez, J,A., Rochina-Barrachina, M.E., Sanchis, A. y Sanchis-Llopis, A. 2017. "The determinants of firms's convergence to the European TFP frontier", Working Papers in Applied Economics, WPAE-2017-07.

Autor, D.H. 2015. "Why are there still so many jobs? The history and future of workplace automation", Journal Economic Perspective, 29(3): 3-30.

Aw, B.Y., Chung, S. y Roberts, M.J. 2000. "Productivity and turnover in the export market: micro-level evidence from the Republic of Korea and Taiwan (China)", The World Bank Economic Review, 14(1): 65-90.

Bartelsman, E., Haltiwanger, J., y Scarpetta S. 2013. "Cross Country Differences in Productivity: The Role of Allocation and Selection”, American Economic Review, 103(1): 305-334.

Benavides Chicón, C. 2012. Calidad y productividad en el sector hotelero andaluz. Universidad de Málaga.

Berlingieri, G., Blanchenay, P. y Criscuolo, C. 2017. "The Great Divergence(s)", CEP Discussion Paper, $\mathrm{N}^{\circ} 1488$.

Bernard, A. B., y Jones, C.I. 1996. "Comparing apples to oranges: productivity convergence and measurement across industries and countries", The American Economic Review, 1216-1238.

Bernard, A.B., Redding, S.J. y Schott, P.K. 2010. "Multiple-product firms and product switching", American Economic Review, 100(1): 70-97.

Bloom, N., Draca, M., y Van Reenen, J. 2015. «Trade Induced Technical Change? The Impact of Chinese Imports on Innovation, IT and Productivity", The Review Of Economic Studies, 83(1): 87-117. doi: 10.1093/restud/rdv039

Brynjolfsson, E., y Yang, S. 1996. Information technology and productivity: a review of the literature. En Advances in computers (Vol. 43, pp. 179-214). Elsevier.

Brynjolfsson, E., y Hitt, L. 1996. "Paradox lost? Firm-level evidence on the returns to information systems spending", Management Science, 42: 541-558.

Brynjolfsson, E., y Hitt, L. 2000. "Beyond Computation: Information Technology, organizational Transformation and Business Performance", Journal of Economic Perspectives, 14: 23-48.

Brynjolfsson, E., y Hitt, L. 2003. "Computing Productivity: Firm-Level Evidence", Review of Economics and Statistics, 85: 793-808.

Buhalis, D., y Law, R. 2008. "Progress in information technology and tourism management: 20 years on and 10 years after the internet - the state of the e-tourism research", Tourism Management, 29(4): 609-623.

Cànoves, G., Forga, J. M. P., y Romero, A. B. 2016. "Turismo en España, más allá del sol y la playa. Evolución reciente y cambios en los destinos de litoral hacia un turismo cultural", Boletín de la Asociación de Geógrafos Españoles, (71): 431-454.

Cassiman, B., Golovko, E., y Martínez-Ros, E. 2010. "Innovation, exports and productivity", International Journal of Industrial Organization, 28(4): 372-376.

Castells, M. 2004. The Information Society. Cheltenham/Northampton (Massachusetts): Edward Elgar.

Cerezo Medina, A., y Guevara Plaza, A. 2015. "El papel estratégico de las tecnologías de la información y las comunicaciones en el turismo", International Journal Of Information Systems And Software Engineering For Big Companies (IJISEBC), 2: 52-69.

Ciccone, A. y Papaioanou, E. 2005. "Human capital, the structure of production, and growth", Working Papers, Universidad Pompeu Fabra, n ${ }^{\circ} 902$.

Coll-Serrano, V., y Blasco-Blasco, O.M. 2009. "Evolución de la eficiencia técnica de la industria textil española en el periodo 1995-2005. Análisis mediante un modelo frontera estocástica”, Estudios de Economía Aplicada, 27(3): 1-32.

Costa, M. T. 2015. La empresa española. En García Delgado, J. L. y Myro, R. (dirs.), Lecciones de Economía Española, Aranzadi, Navarra, pp. 77-89

Cristelli, M., Tacchella, A., y Pietronero, L. 2015. "The heterogeneous dynamics of economic complexity", PloS one, 10(2): 1-15.

Dedrick, J., Gurbaxani, V., y Kraemer, K. 2003. "Information technology and economic performance: a critical review of the empirical evidence", ACM Computing Surveys, 35: 1-28.

De Loecker, J. 2007. "Do exports generate higher productivity? Evidence from Slovenia", Journal of International Economics, 73(1): 69-98.

De Loecker, J. 2013. "Detecting learning by exporting", American Economic Journal: Microeconomics, 5(3): 1-21. 
Delgado-Verde, M., Martín-de-Castro, G., Navas-López, J. E., y Cruz-González, J. 2011. “Capital social, capital relacional e innovación tecnológica. Una aplicación al sector manufacturero español de alta y media-alta tecnología”, Cuadernos de Economía y Dirección de la Empresa, 14(4): 207-221.

Díaz-Chao, Á., y Torrent-Sellens, J. 2010. “¿Pueden el uso de las TIC y los activos intangibles mejorar la competitividad? Un análisis empírico para la empresa catalana”, Estudios de Economía Aplicada, 28(2): 1-24.

Díaz-Chao, A., Sainz-González, J., y Torrent-Sellens, J. 2015. "ICT, innovation, and firm productivity: New evidence from small local firms", Journal of Business Research, 68(7): 1439-1444.

Dreschsler, W., y Natter, M. 2012. "Understanding a firm's openness decisions in innovation”, Journal of Business Research, 65(3): 438-445

Drucker, P. 2012. Management challenges for the 21st century. Routledge.

Esteban Talaya, Á., Millán Campos, Á. y Molina Collado, A. 2000. "La comercialización de productos turísticos en Internet: implicaciones para las agencias de viaje", Cuadernos de Ciencias Económicas y Empresariales, 3: 15-36.

Fernández de Guevara, J. 2011. La productividad sectorial en España: una perspectiva micro. Fundacion BBVA.

Foster, L., Haltiwagner, J., y Krizan, C.J. 1998. "Aggregate Productivity growth: Lessons from microeconomic evidence", NBER Working paper series. $\mathrm{N}^{\mathrm{o}} 6803$

$\mathrm{Fu}$, Ch., y Moral-Benito, E. 2018. "The evolution of Spanish Total Factor Productivity since the global financial crisis", Documentos Ocasionales $\mathrm{N}^{\circ} 1808$.

García-Santana, M., Moral-Benito, E., Pijoan-Mas, J., y Ramos, R. 2016. “Growing like Spain 1995-2007”, Banco de España Working Paper 1609.

Giuri, P., Torrisi, S., y Zinovyeva, N. 2008. "ICT, skills, and organizational change: evidence from Italian manufacturing firms", Industrial and Corporate change, 17: 29-64.

Gómez, A.M. 2011. "Productividad multifactorial del sector manufacturero del: Cauca 1993-2006", Sociedad y Economía, 20: 243-266.

Gómez, V.B. 2015. "Turismo y desarrollo económico en España: del insuficiente reconocimiento a la revalorización de su función estratégica”, Papers de turisme, 14-15: 117-129.

Graetz, G., y Michaels, G. 2017. "Is modern technology responsible for jobless recoveries?", American Economic Review, 107(5): 168-173.

Guerrero, E. I. G., y Rubio, J. L. 2004. "Productividad y tamaño empresarial en la hostelería andaluza: una aproximación*", Cuadernos de CC.EE y EE, 46: 77-94.

Gust, C., y Marquez, J. 2004. "International comparisons of productivity growth: the role of information technology and regulatory practices", Labour economics, 11(1): 33-58.

Haidar, J. I. 2012. "Trade and productivity: Self-selection or learning-by-exporting in India", Economic Modelling, 29(5): 1766-1773.

Helpman, E., Melitz, M.J. y Yeaple, S.R. 2004. "Export versus FDI with heterogenous Firms”, American Economic Review, 94(1): 300-316.

Hjalager, A. M. 2010. "A review of innovation research in tourism”, Tourism management, 31(1): 1-12.

Iacovone, L., y Crespi, G. 2010. "Catching Up with the Technological Frontier: Micro-level Evidence on Growth and Convergence", Industrial and Corporate Change, 19(6): 2073-2096.

Iglesias, P.P., Jambrino, C., y De las Heras, C. 2017. "La innovación en la Pyme: Barreras y facilitadores", Revista de Estudios Regionales, 110: 99-131.

Ito, K., y Lechevalier, S. 2009. "The Evolution of the Productivity Dispersion of Firms: A Reevaluation of its Determinants in the Case of Japan", Review of World Economics, 145(3): 405-429.

Ivars, J.A., Solsona, F.J., y Giner, D. 2015. "Gestión turística y tecnologías de la información y la comunicación (TIC): El nuevo enfoque de los destinos inteligentes", Documents d'Anàlisi Geogràfica, 62(2): 327-346.

Johnson, P. 2018. New firms: An economic perspective. Routledge.

Jorgenson, D. W., y Vu, K.M. 2016. "The ICT revolution, world economic growth, and policy issues", Telecommunications Policy, 40(5): 383-397.

Kumar, R. R., y Singh, M. 2014. "Role of health expenditure and ICT in a small island economy: a study of Fiji”, Quality \& Quantity, 48(4): 2295-2311.

Law, R., Buhalis, D., y Cobanoglu, C. 2014. "Progress on information and communication technologies in hospitality and tourism", International Journal of Contemporary Hospitality Management, 26(5): 727-750. 
Levinsohn, J., y Petrin, A. 2000. "When Industries become more productive, Do firms? Investigating productivity dynamics", NBER Working Paper, 6893: 1-49.

Li, X., y Prescott, D. 2009. Measuring productivity in the service sector. Guelph, Ontario, Canada: Canadian Tourism Human Research Council and University of Guelph.

Lombardi, R. 2019. "Knowledge transfer and organizational performance and business process: past, present and future researches", Business Process Management Journal, Emerald, 25(1): 2-9.

Luintel, K.B., Khan, M., y Theodoridis, K. 2014. "On the robustness of R\&D”, Journal Productivity Analysis, 42(2): 137-155.

Martin de Castro, Alama Salazar, G, Navas López, J.E., y López Sáez, P. 2009. "El capital relacional como fuente de innovación tecnológica”, Innovar. Revista de Ciencias Administrativas y Sociales, 19(35): 119-132.

Medina, A. C., y Plaza, A. G. 2015. "El papel estratégico de las tecnologías de la información y las comunicaciones en el turismo", International Journal of Information Systems and Software Engineering for Big Companies (IJISEBC), 2(2): 52-69.

Melitz, M.J. 2000. Estimating level productivity in differentiated product industries. Mimeo, Harvard University.

Mengistae, T., y Pattillo, C. 2004. "Export orientation and productivity in sub-Saharan Africa". IMF Staff papers, 51(2): 327-353.

Miró Pérez, A.P. y Pereira Sánchez, Á. 2017. "Effects of the Internet use in the technical efficiency of the tourism sector: a case study of the hinterland regions of Catalonia", Pasos, Revista de Turismo y Patrimonio Cultural, 15(3): 561-577.

Miró Pérez, A.P. 2018. "Competitividad, Productividad y TIC. Un Análisis empírico para el sector químico catalán”, Revista Galega de Economía, 27(1): 49-64.

Mohnen, P., y Hall, B. H. 2013.”Innovation and productivity: An update”. Eurasian Business Review, 3(1): 47-65.

Mokyr, J. 2014. "The next age of invention: Technology's future is brighter than pessimists allow", City Journal, 24(1): 12-21.

Navío-Marco, J., Ruiz-Gómez, L.M., y Sevilla-Sevilla, E. 2018. "Progress in information technology and tourism management: 30 years on and 20 years after the internet - Revisiting Buhalis \&amp; Law's landmark study about eTourism”, Tourism Management, 69: 460-470. https://doi.org/10.1016/j. tourman.2018.06.002

O’Mahony, M., y Vecchi, E. 2003. "Is There an ICT Impact on TFP?: A Heterogeneous Dynamic Panel Approach". No. 219. London: National Institute of Economic and Social Research, 2003.

Olley, G., y Pakes, A. 1996. "The Dynamics of Productivity in the Telecommunications Equipment Industry", Econometrica, 64(6): 1263-1297. doi: 10.2307/2171831

Pérez, C. 2010. "Technological revolutions and techno-economic paradigms", Cambridge journal of economics, 34(1): 185-202.

Renda, A., y Dougherty, S. 2016. "Pro-productivity institutions: Learning from national experience", Background paper. OCDE.

Roth, F., y Thum, A.E. 2013. "Intangible Capital and Labor Productivity Growth: Panel Evidence for the EU from 1998-2005", Review of Income and Wealth, 59: 486-508.

Roura, J. R. C., y Morales, J.M.L. 2011. "El turismo: un sector clave en la economía española”, Papeles de economía Española, 128: 2-20.

Sánchez, R. M. 2017. "Una nueva política industrial para España”, Cuadernos de Información económica, 257: 35-47.

Schermelleh-Engel, K., Moosbrugger, H. y Müller, H. 2003. "Evaluating the fit of structural equation models: Tests of significance and descriptive Goodness-of-Fit measures", Methods of Psychological Research online, 8(2): 23-74.

Syverson, C. 2011. "What determines productivity?", Journal Economic Literature, 49(2): 326-365.

Torrent-Sellens, J. 2004. Innovació tecnològica, creixement econòmic i economia del coneixement (pág. 49). Barcelona: Generalitat de Catalunya, Consell de Treball, Econòmic i Social (CTESC).

Torrent-Sellens, J., y Ficapal-Cuí, P. 2010. “TIC, co-innovación y productividad empresarial: evidencia empírica para Cataluña y comparación internacional de resultados/ICT", Revista de economía mundial, 26: 203-233.

Torrent-Sellens, J. 2016. "La economía del conocimiento y el conocimiento de la economía", Oikonomics Revista de Economía, Empresa y Sociedad, 5: 26-32. 
Trequanttrini, R., Shams, R., Lardo, A., y Lombardi, R. 2016. "Risk of an epidemic impact when adopting the Internet of Things: the role of sector-based resistance", Business Process Management Journal, Emerald Group Publishing, 22(2): 403-419.

Tseng, C., y Goo, Y.J. 2005. "Intellectual capital and corporate value in an emerging economy: empirical study of taiwanese manufacturers", $R \& D$ Management, 25: 187-201.

UNCTAD 2008. Measuring the impact of ICT use in business. The case of manufacturing in Thailand. United Nations.

Van der Wiel H, Creusen H, Van Leeuwen G, y Van der Pijll E. 2012. The Dutch productivity performance: cross your border and look around. En: Mas M, Stehrer R (eds.) Industrial Productivity in Europe: Growth and Crisis. Edward Elgar Publishing, Cheltenham, UK, pp 403-428.

Vogel, A., y Wagner, J. 2010. "Higher productivity in importing German manufacturing firms: self-selection, learning from importing, or both?", Review of World Economics, 145(4): 641-665.

Vilaseca, J. y Torrent-Sellens, J. 2005. Cap a l'empresa xarxa. Les TIC i les transformacions de l'empresa catalana. Barcelona: Edicions de la Universitat Oberta de Catalunya.

$\mathrm{Vu}, \mathrm{K}$. M. 2011. "ICT as a source of economic growth in the information age: Empirical evidence from the 1996-2005 period", Telecommunications Policy, 35(4): 357-372.

Wagner, J. 2007. "Exports and Productivity: A Survey of the Evidence from Firm- Level Data", Working Paper $N^{\circ} 4$, University of Lüneburg.

Wu, W., Chan, M, y Chen, C. 2008. "Promoting innovation through the accumulation of intellectual capital, social capital, and entrepreneurial orientation”, R\&D Management, 38: 265-277.

Zayas, J. M. 2015. "La innovación tecnológica en la economía española: la necesidad de un "gran impulso", ICE, Revista de Economía, 883: 109-125. 